\title{
VALORACIÓN LOCAL DEL PATRIMONIO NATURAL PARA EL DESARROLLO TURÍSTICO EN UN TERRITORIO RURAL TRANSFRONTERIZO (SIERRA DEL LAROUCO, GALICIA- NORTE DE PORTUGAL)
}

\author{
Elena De Uña-Álvarez ${ }^{1}, M^{a}$ del Carmen Cuquejo Bello ${ }^{2} \&$ Montserrat Villarino Pérez $^{3}$ \\ Universidad de Vigo y Universidad de Santiago de Compostela
}

\begin{abstract}
RESUMEN
En la mayoría de los espacios rurales de montaña la oferta turístico-recreativa está basada en sus recursos naturales. El conocimiento de la percepción y las expectativas de los actores locales respecto a su valor y potencialidad es indispensable para fomentar un turismo sostenible. La Sierra del Larouco es un espacio transfronterizo que se extiende entre el sur de Galicia (Noroeste de España) y el norte de Portugal. El objetivo principal de la investigación es conocer la percepción, las ideas y las expectativas de sus actores respecto a los bienes disponibles y su utilización como recursos para el desarrollo local a través del turismo. Para ello, han sido realizadas 29 entrevistas a partir de un cuestionario-guía. Este trabajo presenta solo una parte de sus resultados en el contexto de un estudio exploratorio: los que expresan el grado de interés y la valoración que otorgan los actores clave al conjunto de recursos disponibles, entre los cuales el patrimonio natural ocupa una posición destacada; al mismo tiempo, inciden en el potencial que confieren a este patrimonio "propio" como un conjunto de recursos turísticos para favorecer la mejora del ámbito local.
\end{abstract}

Palabras clave: Patrimonio; naturaleza; turismo; montaña; dimensión local.

\section{LOCAL VALUATION OF THE NATURAL HERITAGE FOR TOURISM DEVELOPMENT IN A CROSS-BORDER RURAL TERRITORY (SIERRA DEL LAROUCO, GALICIA-NORTH OF PORTUGAL)}

\section{ABSTRACT}

In most both rural and mountainous spaces the touristic and recreation offer is based on the natural resources. The knowledge of the perception and prospects from the local actors regarding the values and potential of such natural heritage is crucial to promote a sustainable tourism. The Sierra del Larouco is a cross-border rural space, located between the South of Galicia (Spain) and the North of Portugal. The main objective of the research is to know the perception, ideas and prospects of the local actors with respect to the available assets and their use as resources for local development throughout tourism. In order to achieve this objective, 29 interviews have been performed starting from a questionnaire guide. The present work only contains a part of the interviews results because it is an exploratory study:it is related to the degree of interestand valuation of the availableresources expressed by key actors. Among these resources, the natural heritage have a prominent position; at the same time, the results show the potential of the "own"heritage as a set of touristic resources to improve the life conditions in the local dimension.

Key words: Heritage; Nature; Tourism; Mountain; Local dimension.

\footnotetext{
${ }^{1}$ Departamento de Historia, Arte y Geografía. Universidad de Vigo. E-mail: edeuna@uvigo.es

${ }^{2}$ Departamento de Historia, Arte y Geografía. Universidad de Vigo. E-mail: $\underline{\text { mcbello@xunta.edu.es }}$

${ }^{3}$ Departamento de Geografía. Universidad de Santiago de Compostela. E-mail: $\underline{\text { montserrat.villarino@usc.es }}$
}

Fecha de recepción: 17 de mayo de 2017. Fecha de aceptación: 02 de octubre de 2017. 


\section{INTRODUCCIÓN}

La noción del patrimonio ha evolucionado hacia un significado colectivo que enfatiza su construcción desde y para las comunidades humanas. La consideración de los bienes territoriales como elementos del patrimonio surge en la interacción del grado de su conocimiento científico y el discurso que lo define; de la intención de ejercer acciones para su preservación y uso; y de la percepción de las comunidades humanas. Su interés como recurso emerge entre la norma, la cultura y la sociedad. La Sierra del Larouco configura el eje de un territorio de interior transfronterizo, localizado entre el sur de Galicia y el Norte de Portugal (noroeste de la Península Ibérica), que carece de protección normativa. Sin embargo, posee componentes patrimoniales de interés científico, estético, cultural, educativo y socio-económico que resulta necesario valorar.

El turismo es una actividad socioeconómica que está experimentando una continua reorientación interdependiente de los recursos disponibles y de la dinámica de los actores territoriales. El futuro de la mayoría de los territoriostransfronterizos alejados de los espacios urbanos, de carácter ruraly de montaña, está basado en una oferta turística centrada en los componentes de su patrimonio natural y cultural. El sosiego, la belleza paisajística y el contacto con lo auténtico son argumentos en la promoción del uso turístico para revitalizar estos territorios. Pero la conservación y el buen uso del patrimonio turístico integrado en las estrategias de desarrollo local sostenible dependen en gran medida de su reconocimiento y valoración por parte de los actores locales. El objetivo general de este trabajo es realizar un estudio piloto para explorar las ideas y expectativas de los actores locales sobre los recursos disponibles y el turismo en la Sierra del Larouco y su entorno. Los objetivos específicos son realizar una primera aproximación acerca de las ideas sobre la posibilidad de configurar estrategias turísticas para la mejora de las condiciones del territorio y conocer el estado de opinión sobre el papel que podría jugar en tal supuesto el turismo de naturaleza. Presentamos los primeros resultados de la percepción, los conceptos y la predisposición de los actores, obtenidos mediante la realización de entrevistas.Los resultados están referidos solo a una parte de dos bloques de cuestiones de las entrevistas, y su análisis está planteado como un ensayo de carácter exploratorio para confrontar aspectos clave sobre el patrimonio natural en el contexto de una actividad turística aún incipiente.

\section{MARCO CONCEPTUAL}

El patrimonio común define un conjunto de bienes que son compartidos por los colectivos humanos, sin valor como mercancía, reconocidos como lugares de memoria y de vida (LAFUENTE, 2007). El valor de esos bienes está enraizado en la cultura de una comunidad, en su identidad territorial y en sus relaciones sociales. En esta derivación conceptual hacia la noción del patrimonio integrado en el territorioemerge la perspectiva de su valor socioeconómico y cultural, que "está influido por la pertenencia a un determinado entorno territorial" (ORTEGA VALCÁRCEL, 1998: 45).Desde esta perspectiva, el patrimonio define un sistema de elementos materiales e inmateriales más allá de las figuras legales de protección, establecidas por el ámbito institucional. De acuerdo con ALONSO GONZÁLEZ (2014) el valor de los bienes constitutivos del patrimonio común (la naturaleza, la tradición, la cultura y el estilo de vida de una comunidad) emerge al ser considerados como recursos territoriales susceptibles de ser conservados, sostenidos, utilizados y gestionados para determinados fines.Este proceso revela una visión del pasado y del futuro, apoyada en una identidad compartida, y genera una distinción simbólica de los lugares, paisajes y territorios; depende, además, de las interacciones de los actores locales con otros actores de la gobernanza territorial (WEISZ y CLARK, 2011) cuestión que determina en gran medida su nivel de compromiso respecto al patrimonio común. Sobre todo en la dimensión local, como apunta HORLINGS (2015), el concepto de valor posee un significado ligado a lo propio, con un marcado sentido afectivo e instrumental, conectado a la percepción de la singularidad del territorio.

El entendimiento del patrimonio como un conjunto de bienes propios en la dimensión local 
implica que su valoración debe plantearse teniendo en cuenta las ideas y opiniones de los actores territoriales.Por lo tanto, la percepción de la comunidad local sobre los recursos territorialesrefleja el sentido de las relaciones naturaleza-sociedad en el tiempo. La importancia del conocimiento de esa percepción está relacionada con la revitalización de las identidades locales siendo "cada vez más abundantes las propuestas de desarrollo amparadas en la utilización con fines turísticos de los recursos específicos e irrepetibles de cada ámbito" (SILVA PÉREZ y FERNÁNDEZ SALINAS, 2008: 71). La identificación, reconocimiento y toma de conciencia del patrimonio común por parte de los actores locales son esenciales tanto para su preservación como para la planificación de los usos dirigidos hacia un desarrollo sostenible. Su consideración como recurso está conectado a las ideas y las expectativas humanas siendo la valoración humana creadora de ese carácter de recurso (BARRADO TIMÓN, 2011).Lograr la integración delpatrimonio en el turismo para el desarrollo local también es una meta que reposa en intereses compartidos, con un horizonte centrado en la sostenibilidad territorial.

En este contexto, las tendencias del turismo de interioren España (apoyadas en el valor del patrimonio territorial) "vienen apostando por la subjetividad, por lo local o por los microespacios, por un turismo alternativo que permite la búsqueda de lugares fuera de las rutas convencionales" (PILLET CAPDEPÓN, 2012: 361).Entre los objetivos del turismo sostenible definido por la OMT (2013) encontramos los de contribuir al desarrollo local, empoderar a las comunidades locales, mejorar su calidad de vida y mantener la calidad del ambiente físico. El análisis del binomio turismo-sostenibilidad en las directrices propuestas para diversas escalas (CAÑIZARES RUIZ, 2013) pone de manifiesto la importancia del reconocimiento y respeto hacia el patrimonio común para promover un desarrollo autónomo y competitivo de los destinos, mediante modalidades turísticas que revalorizan los recursos locales y representan oportunidades para el reequilibrio territorial. En este sentido, la emergencia del turismo de naturaleza en los territoriosrurales de interior busca en principio la sostenibilidad y el refuerzo de la identidad territorial (LÓPEZ PALOMEQUE y CÀNOVES, 2014; WILLIAMS y LEW, 2015). En estos espacios todavía tiene gran peso su significado de territorio natural o rural que remite al pasado; el aumento de su demanda turística está muy relacionado con esa idea, además de la necesaria diversificación ante los retos del presente en la dimensión global-local (Figura 1). La diversificación de los espacios rurales, teniendo en cuenta su concepto multidimensional (material e inmaterial), es un proceso que supone la reformulación de sus valores y funciones poniendo en valor un conjunto de recursos como referentes del desarrollo local; en este proceso la opción del turismo de naturaleza es una estrategia que puede favorecer la mejora y la sostenibilidad de los territorios de interior. La misma tendencia se observa en el caso de los territorios transfronterizos de interior (HORTELANO MÍNGUEZ, 2015) que tratan de reconfigurar su dinámica territorial como destinos turísticos singulares. Su sostenibilidad está ligada a la responsabilidad individual y colectiva respecto a los recursos disponibles, lo que supone adaptaciones, transformaciones o cambios a largo plazo (SHARPLEY y TELFER, 2015).

Una variable de especial importancia en la dimensión local es la opinión de los actores clave sobre el valor y la contribución del patrimonio común en la configuración de un posible destino turístico (ÁVILA y BARRADO, 2005; VERA et al., 2013; MARTÍNEZ PUCHE, 2013). Este patrimonio conforma un conjunto de recursos que pueden ser transformados en atractivos turísticos (SANCHO y VERA, 2008; NAVARRO, 2015) una vez que los actores territoriales potencian su capacidad para provocar la visita, cuestión que requiere un proceso de reconocimiento, valoración y conversión desde la esfera pública o privada. La percepción de los actores territoriales sobre los recursos turísticos y el turismo deriva de la vivencia, la experiencia y diferentes fuentes de información, integrando las creencias, la historia y la cultura de la comunidad local y ejerciendo una notable influencia en la emergencia de nuevos usos del territorio (TÖRN et al., 2008). Por lo tanto, el análisis de la percepción y la valoración de los actores locales es vital para conocer las preferencias y actitudes de los mismos frente a procesos de desarrollo local que siempre suponen ciertos cambios económicos, socioculturales y políticos. Una detallada revisión de este tema desde la década de los 70 , centrada en el ámbito de las investigaciones turísticas, la proporciona el trabajo de SHARPLEY (2014) quien concluye en la necesidad de abordar estos aspectos desde un enfoque multidimensional de tipo 
exploratorio. CARDONA et al., (2015) plantean asimismo el interés de un enfoque general, partiendo de una hipótesis donde la cultura, sociedad y economía tradicional son determinantes en las actitudes frente al desarrollo turístico.

FIGURA 1

El espacio rural ante los retos del presente

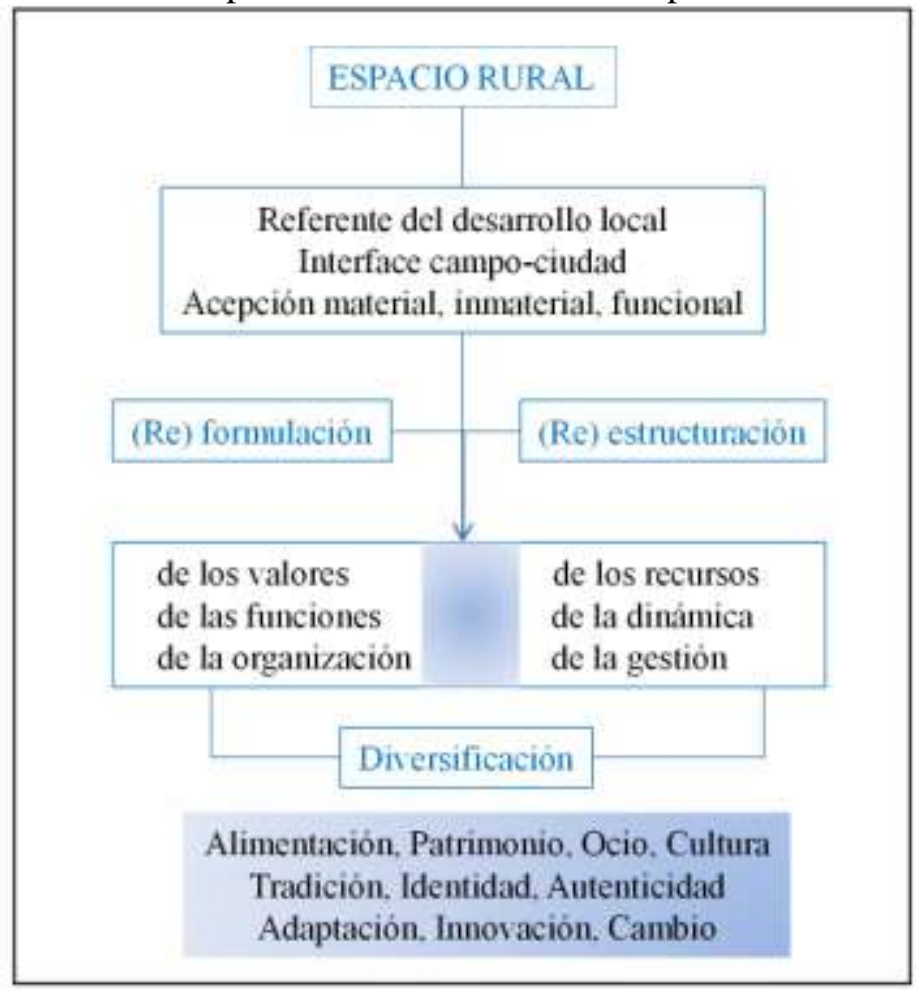

Fuente: Elaboración propia

\section{METODOLOGÍA}

Para abordar el análisis de los aspectos mencionados, a través de la percepción de los principales actores territoriales, la metodología de la investigación siguió tres fases. Durante la primera fase, fue realizado un itinerario completo de la sierra y una visita a los asentamientos del área de estudio. Al mismo tiempo,tuvo lugar la consulta de fuentes cartográficas y bibliográficas, de sitios Web (meteorología, municipios, asociaciones, administración local y autonómica) y de fuentes estadísticas (Institutos Estadísticos Nacional de España, de Galicia y de Portugal) con la finalidad de elaborar un diagnóstico general tanto del área de estudio como del contexto territorial. En la segunda fase, después de identificar a 29 actores del territorio, privados y públicos, que ejercen un papel significativo en el ámbito de los procesos de interés o que pueden proporcionar información cualificada, se elaboró un cuestionario-guía para la recogida de datos. Con cada uno de ellos fue concertada una entrevista (realizada entre 2013 y 2015) que se desarrolló siguiendo la metodología aplicada por CÀNOVES et al. (2014). Durante la tercera fase, las ideas, descriptores y opinionesde las respuestas fueron sometidos a un análisis exploratorio. En este trabajo se presentan los primeros resultados de algunas preguntas pertenecientes a dos bloques de la entrevista, que conciernen específicamente a la percepción y valoracióndel patrimonio común, contemplando las posibilidades de promover el turismo (y la modalidad del turismo de naturaleza),relacionados con las siguientes preguntasguía:

¿Cuáles son, a su entender, los principales valores del territorio y los principales inconvenientes en el momento actual?

¿Podría nombrar tres o cuatro rasgos que definan este territorio hoy?

¿Qué opina sobre las posibilidades del turismo?

¿Cree que el turismo, sobre todo en la naturaleza, mejoraría el desarrollo de la zona? 
Los valores enunciados a partir de la primera pregunta representan la percepción del patrimonio común y de la identidad territorial, mientras que la definición de los inconvenientes representa las dificultades o barreras percibidas. Las respuestas a la segunda pregunta, centrada en los rasgos que definen el territorio, proporcionan un conjunto de descriptores que identifican las ideas e imagen del área de estudio y su entorno. La opinión sobre las posibilidades del turismo, correspondiente a la tercera pregunta, refleja la estimación sobre la potencialidad de los recursos disponibles y la predisposición para el apoyo de su impulso. La última pregunta está planteada con la intención de conocer la percepción de una modalidad turística sostenible respecto a su papel en la mejora de las condiciones del territorio.

El procedimiento de análisis para los resultados de las preguntas se inició con la determinación de las categorías de respuestas y sus frecuencias absolutas, incluyendo los comentarios registrados durante el desarrollo de las entrevistas. La base de datos fue creada utilizando el programa Statgraphics. La codificación de las variables cualitativas en datos ordinales permitió, para el caso de las dos cuestiones que se refieren específicamente al turismo, la aplicación de un análisis estadístico exploratorio. El consenso y grado de acuerdo entre los actores ha sido examinado, a partir del perfil de la muestra, en cuatro grupos de actores: propietarios o gerentes de empresas (código P), administradores o gestores locales (código A), profesionales liberales (código I) y otros informantes cualificados residentes (código R).

\section{CONTEXTO TERRITORIAL}

En el contexto de la Unión Europea, siguiendo la estructura territorial establecida para la gestión de los programas de cooperación, las regiones transfronterizas de montaña son espacios con importantes oportunidades para el desarrollo del turismo debido al valor de sus recursos naturales y culturales pero presentan condiciones socioeconómicas muy dispares (DIJKSTRA y POELMAN, 2011). El logro de los objetivos formulados por la estrategia Europa 2020 (un crecimiento equilibrado, inclusivo y sostenible de las regiones frente a la crisis global) es un reto de particular importancia en los espacios de interior de las regiones transfronterizas periféricas (PAGLIACCI, 2017). Aunque los procesos de diversificación y de transformación basados en los recursos locales son en principio una opción de mejora para ellos, existen dificultades y problemas estructurales que frenan las posibilidades de un desarrollo centrado en la sostenibilidad ambiental, social, económica y cultural. Por ejemplo, TERRES et al., (2015) analizan el abandono del rural en las regiones europeas (implicando el cese del uso y la gestión de los recursos naturales y culturales) y lo explican por razones de tipo multidimensional que incluyen condiciones ambientales hostiles, baja densidad demográfica, envejecimiento, bajo nivel de renta, lejanía de los centros dinámicos y poca capacidad de adaptación. En el mismo sentido, LASANTA et al., (2017) señalan que el abandono de las áreas de montaña europeas ha sido constante desde el siglo XIX afectando especialmente a las de alto valor natural (como es el caso de las del noroeste de España y el norte de Portugal). La pérdida de población y el abandono de tierras en las áreas rurales y de montaña favorecen, además, el incremento del riesgo de incendios; un problema en el que Galicia ocupa la primera posición de España (GÓMEZ-ARMISÉN y ÚBEDA, 2015), siendo preocupante por su incidencia en el norte de Portugal (NUNES et al., 2016).

Dentro de la región transfronteriza conocida como la "raya" entre España y Portugal, el territorio interior del sur de Galicia-Norte de Portugal es predominantemente rural ( $>50 \%$ de la población vive en áreas rurales) y de montaña. La Sierra del Larouco está localizada en este territorio de interior galaico-portugués, alineada en dirección NE-SO, con una extensión superficial de $85 \mathrm{~km}^{2}$ (Figura 2). Su demarcación administrativacomprende dos municipios en el suroeste de la provincia de Ourense, el de Baltar (comarca de A Limia) y el de Cualedro (Comarca de Verín), y la freguesía de Meixedo-Padornelos del municipio de Montalegre (en el norte de la comarca portuguesa de Terras do Barroso). La cota máxima de altitud es la de Pico Larouco $(1.538 \mathrm{~m})$ en Portugal. El territorio de la Sierra del Larouco es un espacio no protegido, entre la Reserva de la Biosfera Gerês-Xurés (al oeste) y el Parque Natural de Montesinho (al este). El paisaje se caracteriza, como en todas las montañas que constituyen este territorio fronterizo, por el dominio de las formas graníticas. La sierra pertenece al contexto de interés 
internacional "Orógeno Varisco Ibérico"(proyecto Global Geosites, IGME, 2014). Los datos meteorológicosmuestran ausencia de confort climático, por déficit de humedad, durante el período estival (julio aseptiembre) y por exceso de frío durante el invierno (diciembre a febrero); las temperaturas medias anuales distinguen el piso bioclimático colino $\left(>10^{\circ} \mathrm{C}\right)$ del montano $\left(6^{\circ} \mathrm{C}-10^{\circ} \mathrm{C}\right)$ mientras que las precipitaciones anuales registradas en los valles (estación del Baltar) son $819 \mathrm{~mm}$ (cumbres). La vegetación original corresponde a bosques de robles pero la mayor parte del espacio forestal de la sierra hoy día está constituido por pinos, introducidos por repoblaciones desde mediados del siglo XX.

\section{FIGURA 2}

Localización y características del área de estudio: a) Panorámica de la sierra desde el suroeste (Montalegre, Portugal); b) Pico Larouco; c) Área de cumbres en la estación de invierno; d) Usos agrarios (Vale da Cerdeira); e) Formas graníticas de interés: Estratificación (pseudobedding).

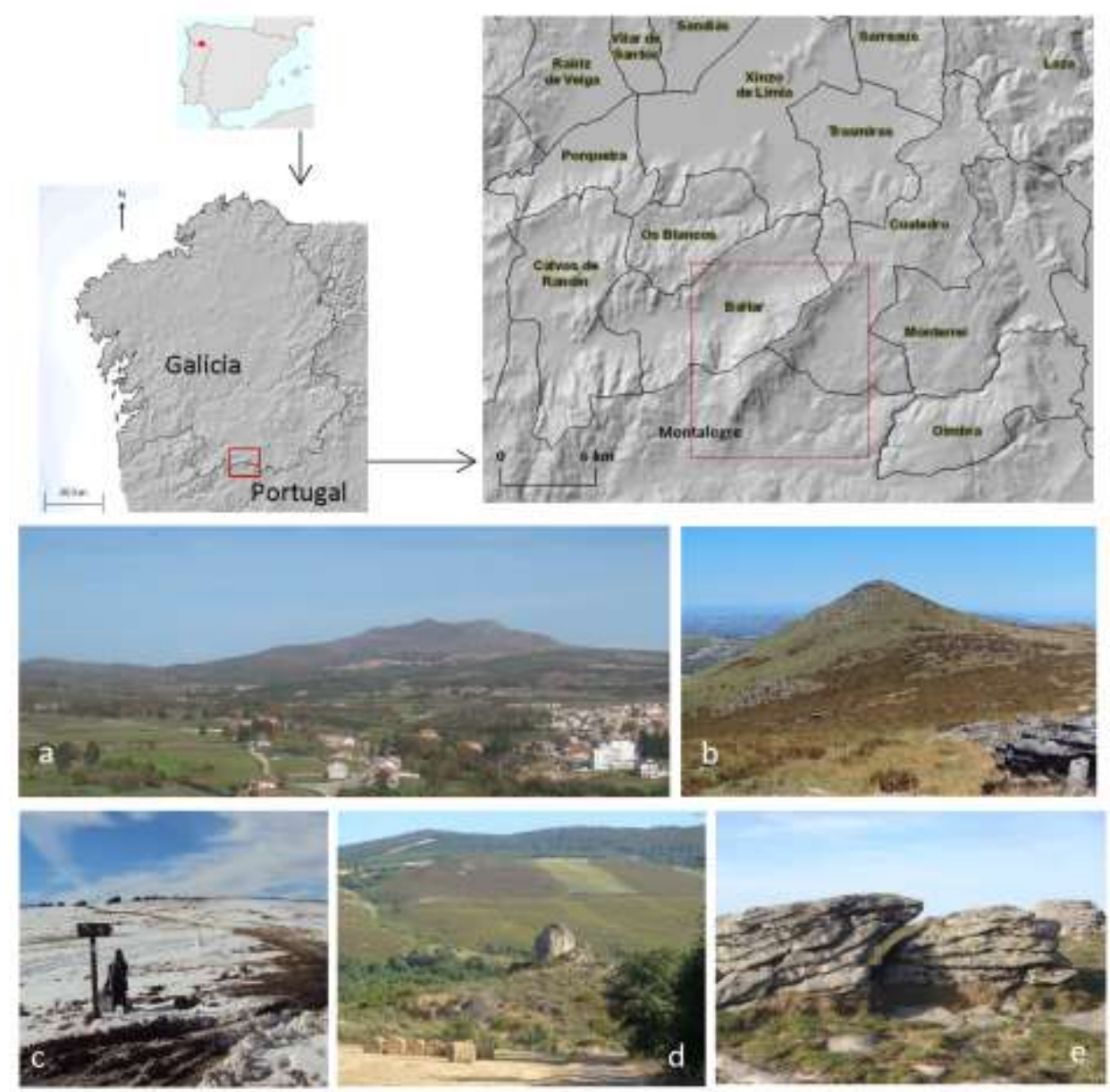

Fuente: Elaboración propia

Los asentamientos humanos, decarácter eminentemente rural, se emplazan en la baja vertiente y los valles, conservandolas viviendas tradicionales. Al comienzo de la década de los 80 los municipios de Baltar y Cualedro contaban con 4.000 y 6.000 habitantes respectivamente, mientras que suman actualmente 986 y 1.792 habitantes (IGE, 2016). En la misma secuencia temporal, la población del municipio de Montalegre pasó de 20.000 a 9.840 habitantes (INEpt, 2016). La densidad demográfica es muy baja: $10 \mathrm{hab} / \mathrm{km}^{2}$ (Baltar), $12 \mathrm{hab} / \mathrm{km}^{2}$ (Montalegre) y $15 \mathrm{hab} / \mathrm{km}^{2}$ (Cualedro). La actividad de la población ocupada está concentrada en el sector servicios $(60 \%)$, con importancia subsidiaria del sector primario $(25 \%)$. Los gestores locales están orientando su estrategia de promoción del territorio hacia la puesta en marcha del turismo de naturaleza pero el proceso es aún incipiente y muy lento. En el municipio portugués de Montalegre se fundó en Ecomuseo de Barrosoen el año 2000, con la finalidad de reforzar la 
identidad local y conjugar la salvaguarda del patrimonio con el impulso del turismo. Un problema común afecta todos los años a los tres municipios de la sierra y su entorno: los incendios forestales. Durante el mes de agosto de 2015 dos incendios tuvieron lugar en el transcurso de una semana. El primero, iniciado en la localidad de Gralhas (Montalegre) arrasó 500 ha en 24 horas en Portugal y se extendió hacia Baltar y Cualedro. El segundo comenzó en el sector NE del Larouco, quemando más 3.200 ha forestales en 48 horas.

\section{ANÁLISIS Y SIGNIFICADO DE LOS RESULTADOS}

\subsection{PERFIL DE LA MUESTRA}

La muestra de los actores entrevistados consta de 19 hombres y 10 mujeres, con edades entre 25 y 70 años. El perfil de los mismos incluye nueve propietarios y gerentes de empresas (grupo P) con edades comprendidas entre 31 y 70 años; cinco administradores y gestores locales (grupo A) con edades entre 25 y 70 años; nueve informantes cualificados que ejercen profesiones liberales (grupo I) con un rango de edad entre 31 y 50 años; y otros informantes cualificados residentes en el área de estudio (grupo R) con edades comprendidas entre 41 y 70 años. Las entrevistas tuvieron lugar en los núcleos de Verín, Cualedro, Baltar, Boullosa y Montalegre.

\subsection{PERCEPCIÓN Y VALORACIÓN DEL PATRIMONIO EN LA DIMENSIÓN LOCAL}

Las respuestas sobre los principales valores del territorio definen los recursos tangibles e intangibles más apreciados por los actores, significativos para la comunidad local y su identidad territorial en el momento actual (Figura 3a). Según el orden de prelación por el número de menciones, destaca el valor de los bienes naturales del territorio (11 menciones) que está asociado a los términos de naturaleza, sierra, aves singulares y medio ambiente; le sigue el valor paisajístico (9 menciones) definido en todas las respuestas como el que corresponde al paisaje de la montaña. El valor como territorio agrícola-ganadero aparece en tercer lugar (8 menciones) definido como un conjunto de recursos y actividades tradicionales que ahora se está perdiendo. Además, se identifican como componentes del valor del territorio (6 menciones) los bienes arqueológicos, históricos y los productos del lugar (por ejemplo miel o carne) ya que reflejan la identidad local, junto con la condición de frontera (4 menciones) a la que se le otorga un valor definido por la expresión "intercambio cultural". Entre los valores intangibles, la percepción de un territorio tranquilo ( 6 menciones) y la belleza del entorno ( 2 menciones) aparecen en relación a su contribución a la buena calidad de vida. El valor turístico del territorio ( 2 menciones) es percibido en relación al elevado potencial para el descanso y el bienestar de las personas con posibilidades de practicar deporte en la naturaleza.

Aplicando los mismos criterios, entre los principales inconvenientes del territorio (Figura 3b) resulta en la primera posición la ausencia de iniciativas emprendedoras (11 menciones); las causas de la escasa motivación para diversificar o innovar las actividades socioeconómicas son definidas por las trabas de la administración pública o las prácticas de caciquismo, poco favorables para la cohesión social. En segunda posición, la carencia de una buena red de infraestructuras (10 menciones) posee una particular importancia que los actores explican por la lejanía de los centros urbanos y el difícil acceso a los servicios sanitarios especializados. El envejecimiento demográfico ( 9 menciones) aparece en tercera posición, relacionado con el despoblamiento (4 menciones), inconvenientes que están conectados en las respuestas al abandono de la actividad agraria, del estilo de vida tradicional y de la vivienda rural. Además, los actores perciben la falta de interés de la administración pública supra-local (5 menciones) y el rigor del clima en la estación de invierno ( 5 menciones) como motivos de la falta de promoción del territorio; estos inconvenientes explican, según las respuestas, el desconocimiento de la sierra y su entorno así como la ausencia de incentivos para atraer a población joven. Los problemas relacionados con los incendios forestales (1 mención) o las canteras (1 mención) apenas tienen representación en los resultados. 
FIGURA 3a

Principales valores:dominiodel patrimonio natural en su acepción material (valor natural), inmaterial (calidad de vida) e integrada (valor paisajístico).

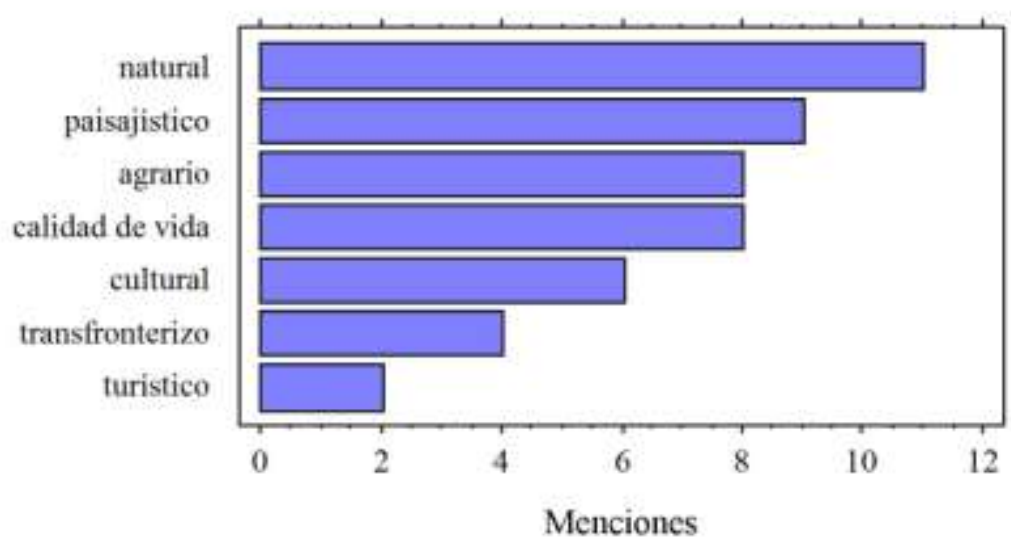

Fuente: Elaboración propia

FIGURA $3 b$

Principales inconvenientes: déficits (ausencia de iniciativas emprendedoras, escasez de infraestructuras, envejecimiento demográfico, falta de interés de la administración supra-local, condiciones del clima) e impactos (incendios, canteras)que obstaculizan el desarrollo local.

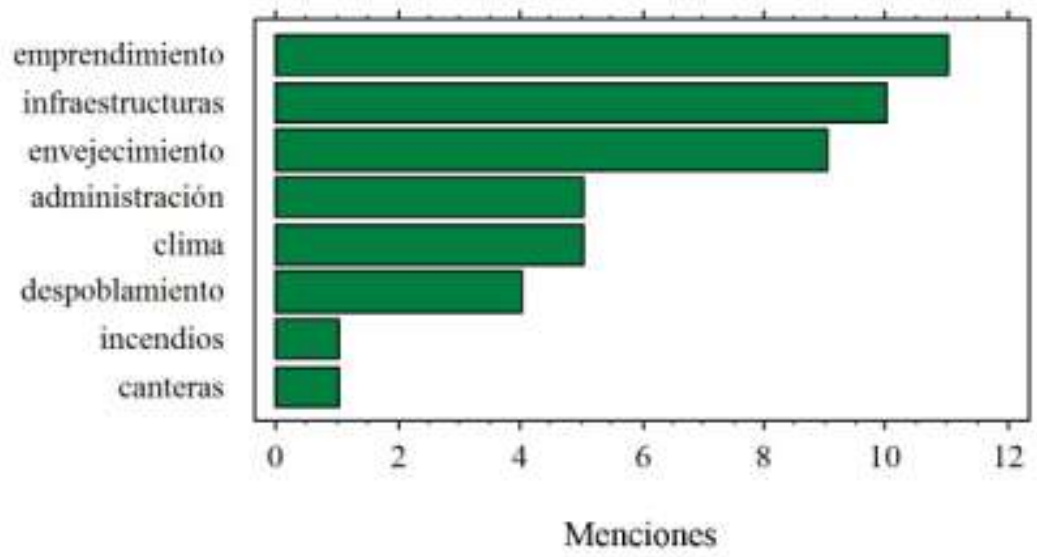

Fuente: Elaboración propia

Los actores entrevistadosenunciancomo rasgos positivos, que definen el territorio, sobre todo los de orden intangible (Figura 4). Las menciones de tranquilidad contienen explícitamente las categorías de sosiego y libertad en la dimensión de la buena calidad de vida; las menciones de la belleza están ligadas a la percepción de la misma respecto a la montaña, el agua, los bienes históricos y los bienes de interés cultural. En la definición de la naturaleza, tercer descriptor por importancia en número de menciones, está incluida la diversidad de los bienes naturales junto con la sierra como término integrador. Con un número de menciones inferior, resulta el descriptor de un ambiente saludable que es definido por el dominio de lo natural, el aire puro y la amabilidad de los residentes. 
FIGURA 4

Ejemplos de descriptores positivos del territorio: a) Tranquilidad y belleza del paisaje, b) Diversidad natural de la sierra, c) Ambiente saludable y deporte, d) Frontera
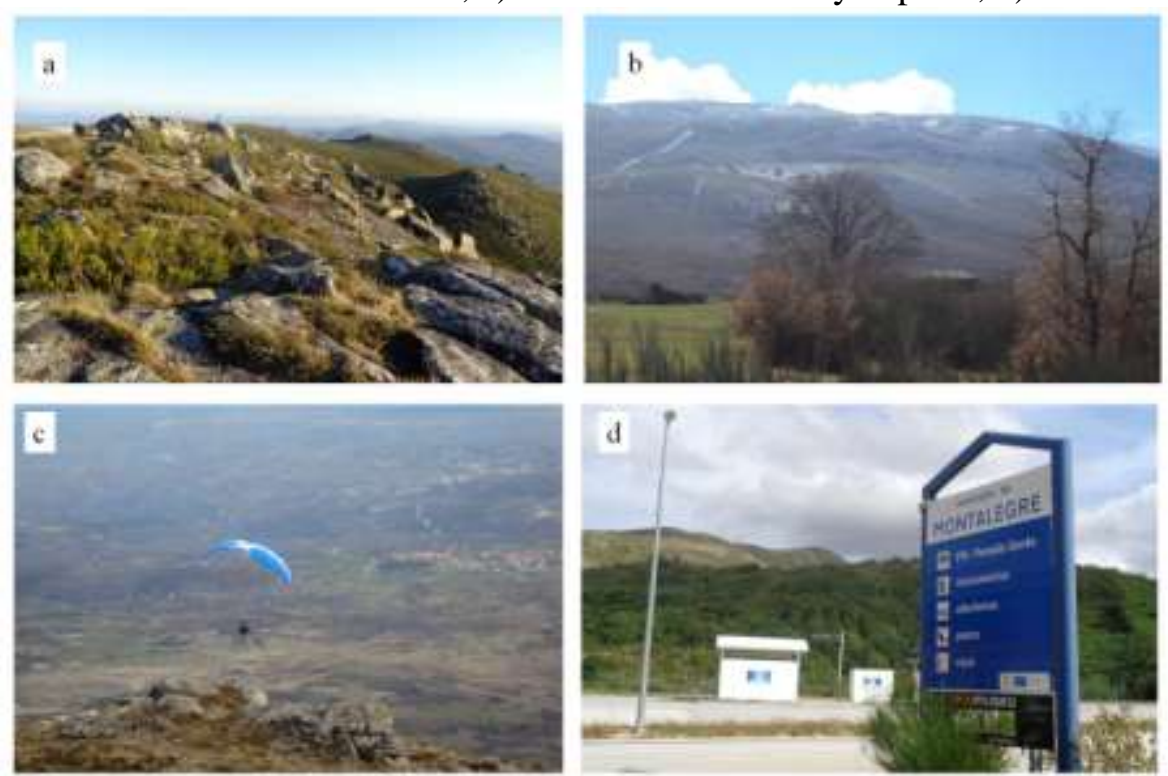

Fuente: Elaboración propia

En la definición de los rasgos positivosestán ausentes la agricultura y la ganadería, siendo el total de menciones mucho más elevado que en el caso de los rasgos negativos (Tabla 1).Los principales rasgos negativos incluidos en la definición del territorio inciden enla difícil situación de los recursos humanos (el envejecimiento demográfico y la despoblación), que los actores relacionan con los procesos de emigración hacia los centros urbanos generando el abandono de las actividades agrarias, la ausencia de oportunidades de mejora y la situación de estancamiento socioeconómico. La jerarquía de categorías que corresponde a los descriptores positivos, por número de menciones, se mantiene en todos los grupos de la muestra de actores por lo que existe entre ellos un alto grado de consenso;en los descriptores negativos existe un menor grado de consenso, revelado por la mayor importancia que los propietarios o gerentes de empresas conceden al envejecimiento demográfico y el abandono del territorio (únicos descriptores mencionados) mientras que solo los profesionales liberales mencionan la deforestación.

TABLA 1

Descriptores de la sierra y su entorno (M=Número de menciones)

\begin{tabular}{|l|c|l|c|}
\hline POSITIVOS & M & NEGATIVOS & M \\
\hline Tranquilidad & 16 & Envejecimiento & 6 \\
\hline Belleza del paisaje y entorno & 14 & Abandono & 6 \\
\hline Naturaleza & 13 & Ausencia de oportunidades & 3 \\
\hline Ambiente saludable & 4 & Baja inversión & 2 \\
\hline Deporte & 1 & Deforestación & 2 \\
\hline Frontera & 1 & Desconocimiento & 1 \\
\hline TOTAL & 49 & TOTAL & 20 \\
\hline
\end{tabular}

Fuente: Elaboración propia

\subsection{POTENCIAL DE LOS RECURSOSY DEL TURISMO}

La mayoría de las respuestas sobre la posibilidad del turismo como posible estrategia de revitalización del territorio incorporan una acción condicional. Los actores que reflejan mayor 
pesimismo opinan que debido a la poca capacidad de inversión y las condiciones socioeconómicas del territorio, las posibilidades de éxito del turismo son muy escasas (1 mención) o escasas (3 menciones). Por lo que respecta a las posibilidades moderadas (3 menciones) o elevadas (18 menciones) los actores condicionan esa situación a la puesta en marcha de iniciativas de uso turístico que no podrían prosperar sin la promoción del territorio. Tan solo quienes opinan que las posibilidades que presenta el territorio para el turismo son muy elevadas (4 menciones) no comentan la necesidad de determinadas condiciones específicas para su desarrollo.Los actores apuntan que en estos momentos existen recursos infrautilizados para la actividad turística: los recursos naturales (naturaleza, agua, montaña) y su aprovechamiento recreativo en modalidades de senderismo o deporte. Las respuestas colocan el senderismo en primer lugar, llamando la atención sobre los recursos que solo tienen un uso turístico ya consolidado en el territorio portugués de la sierra (parapente) aunque se están impulsando en los municipios gallegos. Comentan además el escaso desarrollo del turismo rural, que podría ser promovido vinculado al paisaje y los recursos gastronómicos, y de las rutas turísticas.

Al recodificar estas respuestas en una escala ordinal donde posibilidades muy escasas $=1$, escasas $=2$, moderadas $=3$, elevadas $=4$ y muy elevadas $=5$ (Tabla 2, Figura 5a) la medida de centralidad (mediana) para la opinión de los diferentes grupos de actores es idéntica (posibilidades elevadas del turismo en función de los valores del territorio), manteniendo la misma opinión en el caso de la medida de posición que corresponde al percentil 75 (tercer cuartil); la variabilidad en la opinión sobre este aspecto aparecerelacionada con los rangos de valor del percentil 25 (primer cuartil) donde existe un desacuerdo entre las elevadas posibilidades del turismo (manifestada por los grupos tanto de propietarios o gerentes como de otros informantes cualificadas) y las posibilidades escasas del turismo (manifestada por el grupo de profesionales liberales), si bien resulta minoritaria en el conjunto de la muestra.

TABLA 2

Tendencia central y variabilidad de la opinión sobre las posibilidades del turismo (recodificada en escala ordinal) según los grupos de actores $(\mathrm{G})$.

\begin{tabular}{|c|c|c|c|c|}
\hline G & Rango & Cuartil 1 & Mediana & Cuartil 3 \\
\hline P & $3-5$ & 4 & 4 & 4 \\
A & $2-5$ & 3 & 4 & 4 \\
I & $1-5$ & 2 & 4 & 4 \\
R & $3-4$ & 4 & 4 & 4 \\
\hline
\end{tabular}

Fuente: Elaboración propia

FIGURA 5a

Respuestas sobre las posibilidades del turismo según los grupos de actores

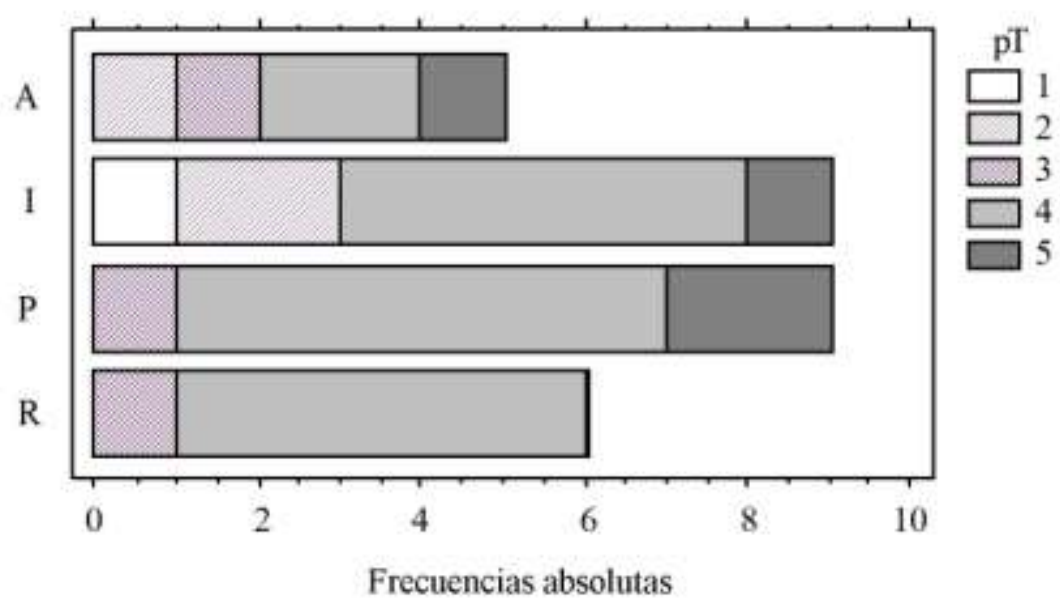

Fuente: Elaboración propia 
La mayoría de las respuestas (17 actores) otorgan un decidido sí, sin condiciones, a la bondad del turismo de naturaleza como agente para la revitalización socio-económica de la Sierra de Larouco y su entorno. La misma opinión aparece condicionada por la necesidad de una promoción adecuada (3actores) que además debe contar con una buena gestión (1 actor) o conuna importante inversión pública y privada para iniciar y mantener a lo largo del tiempo las estrategias de uso turístico (3actores). Para4 actoresel potencial de esta propuesta de uso del territorio solo poseería un carácter complementario de otras actividades (las agropecuarias) que supondrían mayores expectativas de mejora para la comunidad local. Al recodificar estas respuestas en una escala ordinal donde la bondad del turismo de naturaleza se expresa sin condiciones $=5$, con necesidad de promoción $=4$, con promoción y gestión $=3$, con fuerte inversión=2 y quedando restringida al papel complementario de las actividades agrarias $=1$ (Tabla 3, Figura 5b) el grado de desacuerdo entre los grupos de actores es minoritario y queda reflejado en el $25 \%$ de las respuestas (delimitadas por el primer cuartil). En el contexto de las respuestas a esta pregunta durante las entrevistas, los comentarios para los resultados de la escala ordinal con valor 3, 4 y 5 (los actores que presentan una disposición más positiva hacia el potencial del turismo de naturaleza) indicaron como opciones prioritarias el senderismo, las rutas en bicicleta, el turismo micológico y cinegético, y los deportes aéreos. La explicación del alto potencial del turismo de naturaleza para contribuir al avance del territorio está basada en la opinión de su capacidad para detener el abandono de las tierras y la despoblación, favorecer la creación de empleo y la conservación de los recursos naturales, atraer a población joven e impulsar la mejora de las condiciones socioeconómicas.

TABLA 3

Tendencia central y variabilidad de la opinión sobre el potencial del turismo de naturaleza (recodificada en escala ordinal) según los grupos de actores $(\mathrm{G})$.

\begin{tabular}{|c|c|c|c|c|}
\hline G & Rango & Cuartil 1 & Mediana & Cuartil 3 \\
\hline P & $1-5$ & 1 & 4 & 5 \\
A & $1-5$ & 2 & 5 & 5 \\
I & $2-5$ & 4 & 5 & 5 \\
R & $2-5$ & 3 & 5 & 5 \\
\hline
\end{tabular}

Fuente: Elaboración propia

FIGURA $5 b$

Respuestas sobre la potencialidad del turismo de naturaleza para la mejora del territorio según los grupos de actores

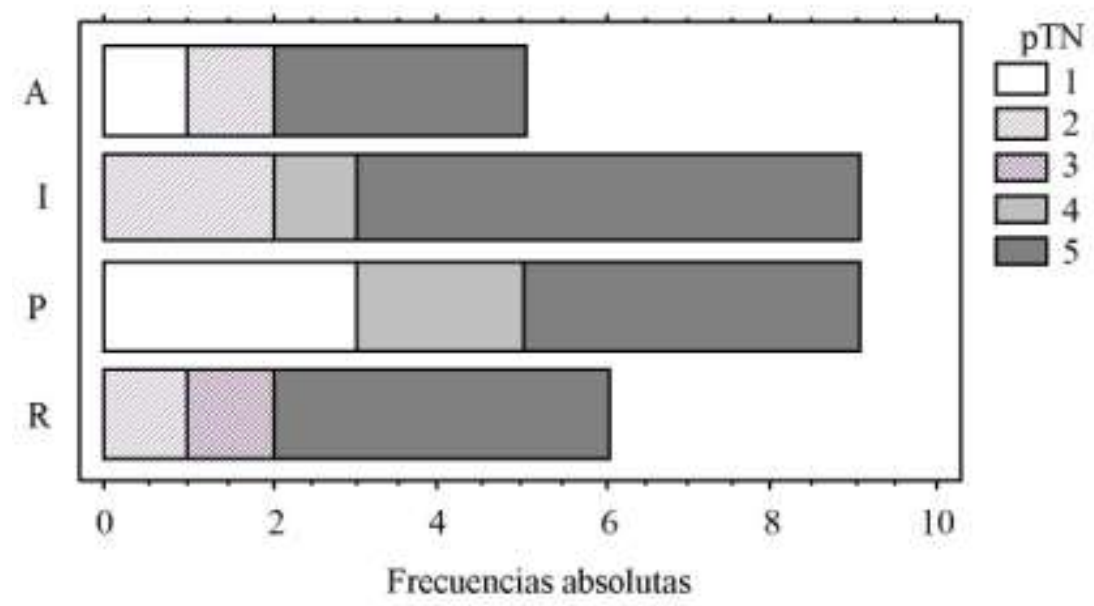

Fuente: Elaboración propia

En la identificación de los principales obstáculos del territorio, que emergen entre los 
condicionantes del impulso del turismo, los resultados de las entrevistas (la percepción del territorio) está de acuerdo con los problemas del abandono y de la despoblación en los territorios rurales y de montaña europeos de los espacios de interior. Sin embargo, la percepción y valoración del problema de los incendios forestales es casi nula, un aspecto a desarrollar en futuras investigaciones y que puede estar relacionado con el hecho de que prácticamente la totalidad de los fuegos son intencionados. Por otra parte, el reconocimiento de los principales valores del territorio, ligados a los atributos tangibles e intangibles del patrimonio natural, también es coherente con la configuración del contexto territorial y de la sierra. Estos valores e inconvenientes sustentan las opiniones sobre las posibilidades de implantar estrategias turísticas y con las opiniones sobre el potencial del turismo de naturaleza para la mejora de la situación del territorio.

\section{CONSIDERACIONES FINALES}

Plantear el futuro de la Sierra del Larouco con una estrategia de desarrollo viable y sostenible es una de las principales preocupaciones revelada en las entrevistas realizadas. La naturaleza y el paisaje, caracterizados por el dominio de las formas graníticas, son los recursos más apreciados entre los actores locales. El espacio de la montaña, sustentado hasta fechas recientes por actividades agro-pecuarias, es percibido como un territorio dotado de valores intangibles (la belleza, la tranquilidad, el descanso) favorable para el desarrollo de las estrategias asociadas al turismo en la naturaleza. Los actores otorgan al patrimonio históricoartístico y otros bienes culturales un valor secundario.

Entre los procesos de dinamización cara a la sostenibilidad, este espacio de interior fronterizo, cuenta con grandes posibilidades para el excursionismo y el turismo en la naturaleza. Sin embargo, en este escenario, fragmentado por el papel de la sierra como límite entre cuencas hidrográficas, estados, comarcas y municipios, la situación del Norte de Portugal presenta mayor competitividad. La situación demográfica es la principal debilidad socioeconómica detectada en el área de estudio. Una posible vía para la mejora y la contribución al equilibrio territorial consiste en la propuesta de revalorizar los bienes naturales con su patrimonio cultural asociado, mediante estrategias conjuntas (transfronterizas)relacionadas con el turismo en la naturaleza, concebidas como alternativas de desarrollo rural sostenible, generadoras de sinergias positivas. Como ha puesto de relevancia la investigación preliminar realizada, la condición crítica de los recursos humanos representa una barrera para su puesta en marcha, conectada a la falta de iniciativas y un bajo grado de innovación en las actividades socio-económicas.

La metodología desarrollada ha permitido un primer conocimiento de la percepción de la comunidad local sobre los recursos disponibles, confirmando los valores positivos que son otorgados a los bienes naturales y la potencialidad del territorio para el turismo de naturaleza. Los actores locales reconocen el valor de los bienes tangibles e intangibles, mostrando aprecio por la pertenencia a un espacio transfronterizo. La mayoría están de acuerdo en la viabilidad del turismo en la naturaleza como propulsor de mejora ambiental y económica del territorio. La percepción y la valoración de los actores es coherente con la orientación turística que existe entre el territorio del sur de Galicia y el Norte de Portugal. En el segundo caso existe una mayor impronta identitaria con gran importancia del turismo deportivo en el medio natural. La investigación continuará en la línea de la interpretación de esta situación territorial, incidiendo en los aspectos clave que han sido determinados por el presente trabajo exploratorio.Debido al carácter preliminar del presente estudio, que solo comprende una parte de las respuestas obtenidas en las entrevistas realizadas y, al mismo tiempo, al hecho de que una investigación completa de mayor profundidad se encuentra en fase de publicación, el análisis presentado responde al enfoque de un estudio piloto. Los resultados y primeras conclusiones del trabajo abren futuras líneas de investigación, que estarán centradas en la integración activa del patrimonio en las estrategias turísticas y en la propuesta de nuevos modos de gestión que contribuyan a la sostenibilidad de los territorios de interior. 


\section{BIBLIOGRAFÍA}

ALONSO GONZÁLEZ, P. (2014): "From a Given to a Construct".Cultural Studies, vol. 28, n 3, p. 359-390.

ÁVILA BERCIAL, R. y BARRADO TIMÓN, D. (2005): "Nuevas tendencias en el desarrollo de destinos turísticos: marcos conceptuales y operativos para su planificación y gestión". Cuadernos de Turismo, ${ }^{\circ} 15$, p. 27-44.

BARRADO TIMÓN, D. (2011): "Recursos territoriales y procesos geográficos: el ejemplo de los recursos turísticos". Estudios Geográficos, vol. LXXII, n 270, p. 35-58.

CÀNOVES, G., VILLARINO, M., BLANCO, A., DE UÑA, E. y ESPEJO, C. (2014): Turismo de interior: renovarse o morir.Servicio de Publicaciones, Universitat de Valencia, col. Desarrollo Territorial, Valencia, $241 \mathrm{pp}$.

CAÑIZARES RUÍZ, M.C. (2013): "Sostenibilidad y Turismo: de la documentación internacional a la planificación en España Horizonte 2020". Boletín de la Asociación de Geógrafos Españoles, n 61, p. 67-92.

CARDONA, J.R., AZPELICUETA, M.C. y SERRA, A. (2015): "Propuesta de componentes generales de las actitudes de los residentes: sociedad tradicional, desarrollo del turismo y evolución de las actitudes". Retur, vol. 9, n 2, p. 240-257.

DIJKSTRA, L. y POELMAN, H. (2011): "Regional typologies: a compilation". European Union Regional Reports, $\mathrm{n}^{\circ}$ 1, p. 1-15.

GÓMEZ-ARMISÉN, N. y ÚBEDA, X.(2015): Wildland Fires - A Worldwide Reality. "Wildfires in Spain: Causes, Evolution and Effects". In Gonçalves, A.J. and Vieira, A.A. (Eds.). Nova Science Publishers. New York, pp. 127-140.

HORLINGS, L.G. (2015): "Values in place; A value oriented approach toward sustainable place-shaping". Regional Studies, Regional Science,vol. 2, n 1, p. 257-274.

HORTELANO MÍNGUEZ, L.A. (2015): "El patrimonio territorial como activo turístico en la raya de Castilla y León con Portugal". Cuadernos de Turismo, n 36, p. 247-268.

IGE. Instituto Galego de Estatística. www.ige.eu [consulta: 19 de Febrero de 2016]

INE. Instituto Nacional de Estadística. www.ine.es [consulta: 19 de Febrero de 2016]

INEpt. Instituto Nacional de Estadística de Portugal. www.ine.pt [consulta: 19 de Febrero de 2016]

LAFUENTE, A. (2007): "Los cuatro entornos del procomún”. Archipiélago: Cuadernos de crítica de la cultura, $\mathrm{n}^{\circ}$ 77/78, p. 15-22.

LASANTA, T., ARNÁEZ, J., PASCUAL, N., RUIZ-FLAÑO, P., ERREA, M.P. y LANARENAULT, N. (2017): "Space-time process and drivers of land abandonment in Europe". Catena, vol. $149, \mathrm{n}^{\circ} 3$, p. 810-823.

LÓPEZ-PALOMEQUE, F. y CÀNOVES, G. (2014): Turismo y territorio. Innovación, renovación y desafios. Tirant Humanidades, Valencia, $735 \mathrm{pp}$.

MARTÍNEZ-PUCHE, A. (2013): Turismos de interior. "Turismo de interior, identidad y cultura glocal. Valoraciones y propuesta”. En Melgosa, F.J. (coord), Síntesis.Madrid, pp. 43-77.

NAVARRO, D. (2015): "Recursos turísticos y atractivos turísticos: conceptualización, clasificación y valoración". Cuadernos de Turismo, nº 35, p. 337-357.

NUNES, A.N., LOURENÇO, L. y CASTRO, A.C. (2016): "Exploring spatial patterns and drivers of forest fires in Portugal (1980-2014)". Science of the Total Environment, $\mathrm{n}^{\circ}$ 573, p. 1190-1202.

OMT (2013): Sustainable tourism for development guidebook. World Tourism Organization, Madrid,225 pp.

ORTEGA VALCÁRCEL, J. (1998): "El patrimonio territorial: el territorio como recurso cultural y económico". Ciudades, vol. 4, p. 33-48.

PAGLIACCI, F. (2017): "Regional path towards Europe 2020 targets: a spatial approach". EuropeanPlanningStudies, vol. 25, nº 4, p. 601-619.

PILLET CAPDEPÓN, F. (2012): "El turismo de interior en la España peninsular: el patrimonio territorial como destino turístico". Boletín de la Asociación de Geógrafos Españoles, $\mathrm{n}^{\circ}$ 59, p. 345-365. 
SANCHO, J. y VERA, J.F. (2008): Turismo en espacios rurales y naturales. Monografías del Atlas Nacional de España, Instituto Geográfico Nacional, Madrid, 363 pp.

SHARPLEY, R. (2014): "Host perceptions of tourism: A review of the research". Tourism Management, vol. 42, no 1, p. 37-49.

SHARPLEY, R. y TELFER, D. J. (2015): Tourism \& Development: concepts and issues. ChannelView, Bristol, USA, 397 pp.

SILVA PÉREZ, R. y FERNÁNDEZ SALINAS, V. (2008): "El patrimonio y el territorio como activos para el desarrollo desde la perspectiva del ocio y del turismo". InvestigacionesGeográficas, $\mathrm{n}^{\circ}$ 46, p. 69-88.

TERRES et al. (2015): "Farmland abandonment in Europe: identification of drivers and indicators, and development of a composite indicator of risk". Land Use Policy, n ${ }^{\circ} 49$, p. $20-$ 34.

TÖRN, A., SIIKAMÄKI, P., TOLVANEN, A., KAUPPILA, P. y RÄMET, J. (2008): “Local People, Nature Conservation, and Tourism in Northeastern Finland".Ecology and Society, vol. 13, n ${ }^{\circ} 1$, p. 1-8.

VERA, J.F., LÓPEZ-PALOMEQUE, F., MARCHENA, M., ANTÓN, S. (2013): Análisis territorial del turismo y planificación de destinos turísticos. Tirant lo Blanch, Valencia, 485 pp.

WEISZ, H. y CLARK, E. (2011): “Society-Nature Coevolution”. GeografiskaAnnaler (B), vol. 93, p. 281-287.

WILLIAMS, S. yLEW, A. (2015): Tourism Geography. Critical understandings of Place, Space and Experience. Routledge, London, 346 pp. 